\title{
A Brief history of the chemical transformation of 2,4,6-trinitrotoluene
}

\author{
(C) Sergey G. Alekseev, Ekaterina K. Petrova, \\ Dmitry N. Kuznetsov, ${ }^{*+}$ and Konstantin I. Kobrakov \\ Department of Organic Chemistry. Russian State University A.N. Kosygin \\ (Technology. Design. Art). Sadovnicheskaya St., 33, Build.1. Moscow, 117997. Russia. \\ Phone: +7 (495)811-01-01. E-mail: occd@mail.ru
}

\begin{abstract}
*Supervising author; ${ }^{+}$Corresponding author
Keywords: conversion, 2,4,6-trinitrotoluene, trotyl, phloroglucinol, methylfloroglucinol, 2,4,6-trihydroxytoluene, dyes, pigments, nitroanilines, coumarins, aurons, pyrocatechols.
\end{abstract}

\begin{abstract}
The results of works published in the open press over the past 100 years on the possibilities of converting one of the most well-known and widely used blasting explosives (EX) - 2,4,6-trinitrotoluene (TNT, TOL, TNT) into "conversion" chemical products are summarized having a diverse and commercially attractive application. Examples of the first industrial use of TNT are not given as explosives, but as inexpensive and affordable chemical raw materials for the production of components of photosensitive compositions. The reasons for the second more intensive period of development of works (late 20th - early 21 st centuries) on revealing the synthetic potential of TNT are noted. The most promising areas of work are discussed: the synthesis of polyfunctional organic compounds of a carbo (hetero) cyclic structure, interesting as biologically active substances, precursors for the synthesis of pharmaceuticals, original polymers and resins, etc. The article makes assumptions about the reasons for the fact that so far TNT has not found wide practical application as an almost universal starting compound for obtaining a wide range of products of small and large-tonnage organic intesa. Using examples of the results of their own work on obtaining original, effective dyes and pigments, sorption-active compounds, structural analogues of hard-to-reach, but practically significant natural substances, the authors suggest ways of involving TNT in the practice of industrial organic synthesis.
\end{abstract}

\section{References}

[1] M. Biryukov. All explosives commander. Weapons. 2016. No.5. P.50-55. (russian)

[2] DE 386433C Verfahren zur herstellung von lichtempfindlichen schichten mit entwicklungskomponenten. Kalle \& CO AG. - Заявлено 07.10.1921. Опубл. 08.12.1923

[3] J. Schmidt, W. Maier. Über licht-empfindliche Diazoverbindungen, I. Mitteil.: Diazo-naphtholsulfonsäure-(1.2.4). Berichte der deutschen chemischen Gesellschaft (A and B Series) 1931. Vol.64. No4. P.767-777. DOI: 10.1002/cber.19310640409

[4] H. Wandrowsky. Die Lichtpaus vefahren. Berlin: Verlag der Papierzictung. 1921. 113p.

[5] DE 422972 Verfahren zur Herstellung lichtempfindlicher Schichten mit Diazoverbindungen / Kalle \& CO AG. Заявлено 28.05.1924. Опубл. 21.06.1927

[6] J.M. Eider. Ausführliches Handbuch der Photographic. W. Knapp. 1927. Vol.4. P.2.

[7] GB 294972A Improvements in the manufacture of diazo-types. Frans van der grinten. Заявлено 03.05.1927. Опубл. 03.08.1928

[8] DE 514084C Verfahren zur Herstellung lichtempfindlicher Schichten, die Diazoverbindungen enthalten / Naamlooze vennootschap chemisc. Заявлено 11.12.1926. Опубл. 12.12.1930

[9] L. Barth, J. Schreder. Ueber die oxydation des resorcins zu phloroglucin. Berichte der deutschen chemischen Gesellschaft. 1879. Vol.12. No.1. P.503-506. DOI: 10.1002/cber.187901201143

[10] A. Calm. Ueber die einwirkung des anilins auf resorcin und hydrochinon. Berichte der deutschen chemischen Gesellschaft. 1883. Vol.16. No.2. P.2786-2814. DOI: 10.1002/cber.188301602234

[11] H. Gilman. Organic Syntheses. New York: John Wiley \& Sons. 1941. 1st ed. 144p.

[12] H. Gilman. Organic Syntheses. New York: John Wiley \& Sons. 1941. 1st ed. 1526p.

[13] P. Karrer. Organic Chemistry. New York: Nordemann, Publishing Co. 1938. 402p.

[14] L. Barth, J. Schreder. Ueber die einwirkung von schmelzendem aetznatron auf phenol und die synthese des phloroglucins. Berichte der deutschen chemischen Gesellschaft. 1897. Vol.12. No.1. P.417-423. 
DOI:10.1002/cber.187901201114

[15] F. Blau. Versuche über die einwirkung von natriummethylat auf einige brombenzole. Monatshefte für Chemie. 1886. Vol.7. P.621-636. DOI: 10.1007/BF01516602

[16] A.Y. Heintzel. Abhandlung über triaminophenol. Journ. Prakt. Chem. C. 1867. P.375-379.

[17] E. Flesch. Über eine neue synthese des phloroglucins. Monatshefte für Chemie. 1897. Vol.18. P.755-763.

[18] H. Weidel, J. Pollak. Zur kenntniss der nitrosoderivate der phloroglucinäther. Monatshefte für Chemie. 1900. Vol.21. P.15-35.

[19] H. Weidel, J. Pollak. Über die nitrosirung des methylphloroglucins. Monatshefte für Chemie. 1990. Vol.21. P.50-61.

[20] M.L. Kastens, Y.F. Kaplan. TNT into phloroglucinol. Industrial and Engineering Chemistry. 1950. Vol.42. No.3. P.402-413. DOI: 10.1021/ie50483a009

[21] Actual problems of the disposal of missiles and ammunition. Safety, resource saving, ecology. 10th Anniversary International Scientific and Practical Conference. Collection of reports. Ulan-Ude. 2015. 232p. (russian)

[22] V.A. Tartakovsky, S.A. Shevelev, M.D. Dutov. Problems of trotil (TNT) processing into condensation monomers, polymers and dyes in "Conversion Concept for Commercial Applications and Disposal. Technologies of Energetic Systems” Ed. H.Krause Dordrecht, Kluwer Academic Publishers. 1997. P.137.

[23] V.A. Tartakovsky, S.A. Shevelev, A.L. Rusanov. TNT a substance for destruction or a molecule for creation?: thesis. Doc. 16th Mendeleev Congress on General and Applied Chemistry. Section. Status and development of the production of chemical products. Materials of the future and non-traditional chemical energy sources. Moscow. 1998. P.504. (russian)

[24] S.A. Shevelev, V.A. Tartakovsky, A.L. Rusanov. Conversion of demilitarized TNT to higher value products. International Journal of Energetic Materials and Chemical Propulsion. 2002. Vol.5. P.62-70. DOI: 10.1615/IntJEnergeticMaterialsChemProp.v5.i1-6.90

[25] V.A. Tartakovsky, S.A. Shevelev, A.L. Rusanov. 2,4,6-Trinitrotoluene (trotyl) as a multipurpose chemical raw material: abstract. doc. 4th International conference on internal ballistics and combustion processes in solid propulsion systems and guns (ICOC 2002). Russia. Moscow. 2002. P.45-49. (russian)

[26] $R U$ 2292329. The method of obtaining 2,4,6-trihydroxytoluene. S.A. Shevelev, A.Kh. Shahnes, S.S. Vorobiev; applicant Institute of Organic Chemistry named after N.D. Zelinsky RAS. Announced 12.10.2005. Publ. 27.01.2007 (russian)

[27] $R U$ 2233267. $\mathrm{N}^{1}, \mathrm{~N}^{1}, \mathrm{~N}^{2}, \mathrm{~N}^{2}, \mathrm{~N}^{3}, \mathrm{~N}^{3}$-Hexa (2-hydroxypropyl) -2,4,6-triaminotoluene and a method for its preparation. S.I. Orlov, S.V. Popkov, V.L. Grishin, T.S. Serebryakova, A.L. Alekseenko, D.I. Lyamkin, S.A. Shevelev, M.D. Dutov; applicant Institute of Organic Chemistry named after N.D. Zelinsky RAS. Announced 26.11.2002. Publ. 27.07.2004 (russian)

[28] RU 2396245. The method of obtaining 1,3-diamino-5-nitrobenzene. V.A. Kashaev, G.Kh. Khisamutdinov, A.P. Bavrina, S.I. Valeshniy, V.P. Ilyin, N.V. Sedova; applicant FSUE GNII "Crystal". - Announced 29.12.2008. Publ. 10.08.2010 (russian)

[29] K.I. Kobrakov, G.S. Stankevich, A.G. Ruchkina, O.V. Volyansky, O.V. Kovalchukova, A.I. Alafinov. A new intermediate for promising azo dyes based on 2,4,6-trihydroxytoluene. Technology of the textile industry. 2012. No.3. P.142-144. (russian)

[30] M.A. Bastrakov, A.M. Starosotnikov, M.A. Leontieva, A.Kh. Shakhnes, S.A. Shevelev. Synthesis of polycyclic indole derivatives from 2,4,6-trinitrotoluene. Meendeleev Commun. 2009. No.1. P.47-48. DOI:10.1016/j.mencom.2009.01.019

[31] A.L. Rusanov, L.G. Komarova, D.Yu. Likhatchev, S.A. Shevelev, V.A. Tartakovsky. Condensation monomers and polymers based on 2,4,6-trinitrotoluene. Russ chem rev. 2003. Vol.72. No.10. P.899-911.

[32] DOI: 10.1070/RC2003v072n10ABEH000850

[33] O.Yu. Sapozhnikov, V.V. Mezhnev, M.D. Dutov, S.A. Shevelev. Nucleophilic addition to the double bond of 2,4,6-trinitrostyrene and transformations of adducts. Russian Chemical Bulletin. 2005. Vol.54. P.1048-1051. DOI: 10.1007/s11172-005-0357-6

[34] G. Zlotin, G. Kislitsin, V. Samet, A. Serebryakov, D. Konyushkin, V. Semenov, A. Buchanan, A. Gakh. Synthetic utilization of polynitroaromatic compounds. 1.S-Derivatization of 1-substituted 2,4,6trinitrobenzenes with thiols. J. Org. Chem. 2000. Vol.65. No.25. P.8430-8438.

[35] O.V. Serushkina, M.D. Dutov, S.A. Shevelev. Regioselective replacement of nitro groups in 2,4,6trinitrotoluene under the action of alkanethiols. Systhesis of ortho-(alkylthio)-substituted nitrotoluenes and their oxidation to sulfoxides and sulfones. Russian Chemical Bulletin. 2001. Vol.50. Iss.2. P.261-264.

[36] V.V. Rozhkov, A.M. Kuvshinov, S.A. Shevelev. Interatction of 2,4,6-trinitrotoluene and its analogues with aldehydes, synthesis of benzoannelated heterocycles from the products of condensation. Synt. 
Commun. 2002. Vol.32. No.9. P.1465-1474. DOI: 10.1081/SCC-120003645

[37] A.L. Rusanov, L.G. Komarova, M.P. Prigozhina, A.A. Askadskii, S.A. Shevelev, A.Kh. Shakhnes, M.D. Dutov, O.V. Serushkina. New fluorinated polyimides. Polym. Sci. Ser. B. 2006. Vol.48. P.209212. DOI: $10.1134 / \mathrm{S} 1560090406070141$

[38] $R U P \Phi 2285015$. A method of obtaining a phosphorus-containing triaminotoluene-formaldehyde resin and its use as a component of fire retardant coatings. A.M. Sakharov, S.P. Krukovsky, A.A. Yarosh, M.Yu. Popovich, Institute of Organic Chemistry N.D. Zelinsky RAS. Announced 27.09.2005. Publ. 10.10.2006 (russian)

[39] S.N. Chukova, A.Kh. Shakhnes, N.S. Perov, V.G. Krasovskii, S.A. Shevelev, A.A. Kuznetsov. 2,4,6Tri(4-aminophenoxy)toluene and hyperbranched polyimide derived from it. Russian Chemical Bulletin. 2015. Vol.64. No.2. P.473-474. DOI: $10.1007 / \mathrm{s} 11172-015-0890-\mathrm{x}$

[40] A.A. Kuznetsov, S.N. Akimenko, A.Y. Tzegel'skaya, N.S. Perov, G.K. Semenova, A.Kh. Shakhnes, S.A. Shevelev. Synthesis of branched polyimides based on 9,9-bis(4-aminophenyl)fluorene and an oligomeric trianhydride, a 1,3,5-triaminotoluene derivative. Polym. Sci. Ser. B. 2014. Vol.56. P.41-48. DOI: $10.1134 / \mathrm{S} 1560090414010060$

[41] F. Yuste, R. Sánchez-Obregón, F. Walls. The synthesis of grandinol. Tetrahedron Letters. 1978. Vol.19. No.49. P.4869-4870.

[42] T. Matsumoto, Inder Pal Singh, Hideo Etoh, Hitoshi Tanaka H. The First total synthesis of grandinal, a new phloroglucinol derivative isolated from eucalyptus grandis. Chemistry Letters. 2001 Vol.30. No.3. P.210-211. DOI: $10.1246 / \mathrm{cl} .2001 .210$

[43] G.R. Ramage, W.J.I. Stowe. The structure and synthesis of bæckeol. J. Chem. Soc. 1940. P.425-426. DOI: $10.1039 / J R 9400000425$

[44] GB 584731A Manufacture of new azo-dyestuffs. CHEM IND BASEL. Заявлено 10.08.1942. Опубл. 22.01.1947

[45] $R U$ 2102415. Mono- and disazo derivatives based on 2,4,6-triaminotoluene as azo dyes and methods for their preparation. V.V. Shaburov, B.A. Lebedev, I.V. Krauklish, A.A. Astratiev, T.V. Artamonova. Announced 30.09.1994. Publ. 20.01.1998 (russian)

[46] J. Callum, Me Hugh, R. Keir, D. Gahan, W.E. Smith. Selective functionalization of TNT for sensitive detection by SERRS. Chem.Comm. 2002. No.6. P.580-581. DOI: 10.1039/B110972C

[47] S.A. Shevelev, A.Kh. Shakhnes, B.Y. Ugrak, S.S. Vorob'ev. Highly selective one-step synthesis of 2amino-4,6-dinitrotoluene and 2,6-diamino-4-nitrotoluene from 2,4,6-trinitrotoluene. Synthetic Commun. 2001. Vol.31. P.2557-2561. DOI: 10.1081/SCC-100105379

[48] Singh Inder Pal, Jasmeen Sidana, S.B. Bharate, W.J. Foley. Phloroglucinol compounds of natural origin: Synthetic aspects. Natural Product Reports. 2010. Vol.27. No.3. P.393-416. DOI: 10.1039/B914364P

[49] O.P. Grukova, G.S. Stankevich, K.I. Kobrakov, A.Kh. Shakhnes, S.A. Shevelev. Synthesis of azo dyes from 2,4,6-trinitrotoluene and 1,3,5-trinitrobenzene. Chemical Technology. 2003. No.7. P.12-19. (russian)

[50] RU 2273652. Dyes based on 3,5-dinitroaniline and 2-amino-4,6-dinitrotoluene. O.P. Grukova, G.S. Stankevich, K.I. Kobrakov, MSTU A.N. Kosygin. Announced 30.12.2004. Publ. 10.04.2006 (russian)

[51] K.I. Kobrakov, D.N. Kuznetsov, A.G. Ruchkina, N.M. Sharpar. Synthesis and properties of azo compounds based on nitroanilines - derivatives of 2,4,6-trinitrotoluene and 1,3,5-trinitrobenzene. Chemical Technology. 2019. Vol.20. No.10. P.440-444. (russian) DOI: 10.31044 / 1684-5811-2019-20$10-440-444$

[52] V.I. Ushkarov. Synthesis and study of the properties of azo compounds based on methylphloroglucinol: $\mathrm{PhD}$ : 02.00.03. Moscow. 2006. 140p.

[53] A.I. Alafinov. Synthesis and study of the properties of methylfloroglucin derivatives: PhD: 02.00.03. Moscow. 2013. 135p.

[54] V.I. Ushkarov, K.I. Kobrakov, A.I. Alafinov, S.A. Shevelev, A.Kh. Shakhnes. Methylphloroglucinol as an available semiproduct for azo dye synthesis. Theor Found Chem Eng. 2007. Vol.41. P.671-674. DOI: $10.1134 / \mathrm{S} 0040579507050375$

[55] V.I. Ushkarov, K.I. Kobrakov, A.I. Alafinov, G.S. Stankevich, S.A. Shevelev, A.Kh. Shakhnes. Methyl phloroglucinol-based dyes for chemical fibres. Fibre Chemistry. 2006. Vol.38. No.3. P.188-190. DOI: 10.1007/s10692-006-0067-3

[56] A.I. Alafinov, K.I. Kobrakov, D.N. Kuznetsov, and M.B. Dmitrieva. Synthesis of new nitrogen of methyl phluoroglucinol - potential dyes and pigments for textiles. Butlerov Communications. 2013. Vol.33. No.3. P.93-99. ROI-jbc-02/13-33-3-93

[57] $R U 2462544 C 1$. The method of cold dyeing with insoluble azo dyes of fibrous materials. Tarakanova 
L.I., Safonov V.V., Kobrakov K.I., Stankevich G.S. MSTU A.N. Kosygin. Announced 08.04.2011. Publ. 27.09.2012 (russian)

[58] Pinto, Madaleno M., Polonia, Joaguim. Synthesis of new xanthones. Helr. Ohim. Acta. 1974. Vol.57. No.8. P.2613-2617.

[59] M. Lrounasmaa, C.Y. Widen, C.M. Tunf, A. Huhtikanagas. On the phloroglucinol derivatives of Mallotus philippinensis. Planta Med. 1975. Vol.28. No.1. P.16-31. DOI: 10.1055/s-0028-1097825

[60] B.S. Joshi, D.H. Gawad. Flavanones from the stem of Unona Lawil. Ysolation of lawinal, desmethoxymafteucinol, desmethoxymafteucinol-7-methyl ether and a synthesis of lawinal. Jndian J. Chem. 1974. Vol.12. No.10. P.1033-1037.

[61] Radhakrishna K.M. Pillai, Prakash Naiksatam, Francis Johnson, Raghavan Rajagopalan, Paul C. Watts, Renato Cricchio, Sonia Borras Thermorubin II. 1,3-Dihydroxy-9H-xanthones and 1,3-dihydroxy-9Hxanthenes. New methods of synthesis. J. Org. Chem. 1986. Vol.51. No.5. P.717-723. DOI: 10.1021/jo00355a024

[62] Rama I. Mani, Parris F. Powers, Landson Drummond Synthesis of flavonols analogous to guercetin employing the Baker-Venkataraman reaction. J. Tenn. Acad. Sci. 1993. Vol.68. No.3. P.83-86.

[63] S.S. Bobylev, K.I. Kobrakov, D.N. Kuznetsov, A.G. Ruchkina, S.A. Shevelev, A.K. Shakhnes, A.N. Fakhrutdinov. Synthesis of 5,7-dihydroxy-4,8-dimethylchromen-2-one and its azo derivatives. Russian Chemical Bulletin. 2015. Vol.64. No.1. P.154-160. DOI: 10.1007/s11172-015-0836-3

[64] S.S. Bobylev, K.I. Kobrakov, D.N. Kuznetsov, A.G. Ruchkina, A.N. Fakhrutdinov. Synthesis and transformations of dihydroxy-2H-1-benzopyran-2-ones. Russian Journal of Organic Chemistry. 2015. Vol.51. No.11. P.1572-1577. DOI: 10.1134/S1070428015110093

[65] D.A. Shubin, D.N. Kuznetsov, K.I. Kobrakov, A.M. Starosotnikov, N.Y. Merkulova. Synthesis of aurone derivatives on the basis of 2,4,6-trihydroxytoluene. Chem. Hetorocycl. Compd. 2019. Vol.55. No.12. P.1174-1178. DOI: 10.1007/s10593-019-02597-0

[66] V.V. Melyshenkova, D.N. Kuznetsov, A.G. Ruchkina, K.I. Kobrakov. Synthesis of new acridine-9carboxylic acid derivatives. Russian Chemical Bulletin. 2018. Vol.67. No.5. P.878-883. DOI: 10.1007/s11172-018-2152-1

[67] D.A. Shubin, S.S. Bobylev, D.N. Kuznetsov, A.G. Ruchkina, K.I. Kobrakov. Synthesis and some properties of 2,4,6-trihydroxy-3-methylbenzoic acid. Russian Chemical Bulletin. 2019. Vol.68. No.1. P.74-78. DOI: $10.1007 / \mathrm{s} 11172-019-2418-2$

[68] V.N. Lisitsin. Chemistry and technology of aromatic compounds. Moscow: De Lee plus. 2014. 390p. (russian) 